\title{
Enhanced Hanbury Brown and Twiss interferometry using parametric amplification
}

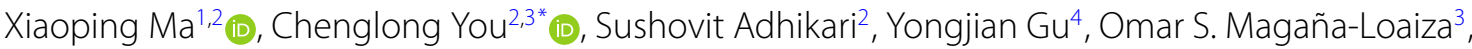 \\ Jonathan P. Dowling $2,5,6^{\wedge}$ and Hwang Lee ${ }^{2}$
}

\author{
"Correspondence: cyou2@lsu.edu \\ ${ }^{2}$ Hearne Institute for Theoretical \\ Physics and Department of Physics \\ \& Astronomy, Louisiana State \\ University, Baton Rouge, USA \\ ${ }^{3}$ Quantum Photonics Laboratory, \\ Department of Physics and \\ Astronomy, Louisiana State \\ University, Baton Rouge, USA \\ Full list of author information is \\ available at the end of the article \\ Deceased
}

\begin{abstract}
The Hanbury Brown and Twiss (HBT) interferometer was proposed to observe intensity correlations of starlight to measure a star's angular diameter. As the intensity of light that reaches the detector from a star is very weak, one cannot usually get a workable signal-to-noise ratio. We propose an improved HBT interferometric scheme incorporating optical parametric amplifiers (OPA) into the system to amplify the correlation signal. Remarkably, for weak star light, the signal-to-noise ratio (SNR) in the new HBT interferometric scheme is much better than that of conventional HBT interferometer. Our work is valuable in measuring a star whose intensity at the detector is low and maybe also applicable in remote sensing and long-distance quantum imaging where the light passed through the object is weak after a long distance transmission.
\end{abstract}

Keywords: HBT; OPA; SNR

\section{Introduction}

The angular diameter of stars, which is a fundamental property estimated in all models of stellar evolution, is important for the determination of other stellar properties including fluxes, effective temperatures, radii and absolute luminosities, and for providing constrains on theoretical stellar models [1]. The Hanbury Brown and Twiss (HBT) interferometer is one tool initially developed to measure the angular size of stars.

In 1956, HBT interferometer, a type of optical interferometer, was reported by Hanbury Brown and Twiss to measure correlations in intensity fluctuations [2, 3]. For over half a century, the HBT interferometer has played a crucial role in understanding the nature of light by motivating both theoretical and experimental research on the coherence properties of optical fields [4-10]. Moreover, besides the study of optical fields, the HBT interferometer has also been applied in other physical systems. Baym discussed the basic physics of intensity interferometry and its application in high energy nuclear physics, condensed matter physics and atomic physics [11]. Jeltes et al. studied differences between fermionic and bosonic HBT interferometers [12]. Recently, Campagnan et al. introduced

(c) The Author(s) 2020. This article is licensed under a Creative Commons Attribution 4.0 International License, which permits use sharing, adaptation, distribution and reproduction in any medium or format, as long as you give appropriate credit to the original author(s) and the source, provide a link to the Creative Commons licence, and indicate if changes were made. The images or other third party material in this article are included in the article's Creative Commons licence, unless indicated otherwise in a credit line to the material. If material is not included in the article's Creative Commons licence and your intended use is not permitted by statutory regulation or exceeds the permitted use, you will need to obtain permission directly from the copyright holder. To view a copy of this licence, visit http://creativecommons.org/licenses/by/4.0/. 
a HBT interferometer realized with anyons, which can directly probe entanglement and fractional statistics of initially uncorrelated particles [13]. Other interesting applications can be found in Refs. [14-21].

In this paper, we will discuss how to improve the HBT interferometer system if the original purpose on measuring the angular diameter of stars is retained. The HBT interferometer was utilized for the first time to determine the angular diameter of Sirius. This star was particularly chosen because it was the only star bright enough to give a workable signal-to-noise ratio (SNR) [2]. Now the question arises, is it possible to apply HBT interferometry for stars that are much less brighter than Sirius? We answer this question in the affirmative by proposing an interferometric scheme that utilizes optical parametric amplifiers (OPAs) to boost the SNR of HBT interferometers. Specifically, our idea is to amplify the signal (starlight) before it is passed into the correlator by using two OPAs. The OPAs boost the input photon number, the effective intensity of the measured star light, and ultimately the correlation signal amplitude of the HBT interferometer. Although the noise also increases, the increase in noise is lower than that of signal. The net effect leads to an amplified SNR in the HBT interference structure. Our result shows that, for the case of weak starlight (or the mean photon number is very low), the SNR is much better than that of conventional HBT interferometer. Thus, the new HBT interferometric scheme is helpful for measuring the angular diameter of stars whose intensity at the detector is low. For example, Vega, the second brightest star in the night sky, has $0.95 \times 10^{-4}$ photons per unit optical bandwidth per unit area and unit time at $443 \mathrm{~nm}$ as the flux [22]. Using our interferometric scheme to measure the angular diameter of Vega, the SNR could be increased by two orders of magnitude. Furthermore, our idea using OPAs also can be used in other quantum tasks, such as long-distance quantum imaging, where optical signal containing object information will decay in a long-distance transmission through the air.

\section{The HBT interferometer}

The HBT interferometer is an extension of the Michelson stellar interferometer. The Michelson stellar interferometer measures the correlation of the electric field in order to measure the angular diameter of the stars, whereas, the HBT interferometer measures intensity correlations of light to measure the star's angular diameter.

The basic idea behind the HBT interferometer is shown in Fig. 1. Let $\vec{k}$ and $\vec{k}^{\prime}$ be the wave vectors of two light beams produced by independent sources on the disc of a star and $\phi$ be the angle between the emitted light. Assuming, sources $\vec{k}$ and $\vec{k}^{\prime}$ produces electric fields

Figure 1 Schematic diagram of a HBT interferometer. $\vec{k}$ and $\vec{k}^{\prime}$ are the wave vectors of the two rays. The intensities are measured at $r_{1}$ and $r_{2}$ by two detectors and the signals are combined in an electronic correlator, which calculates the second order correlation function

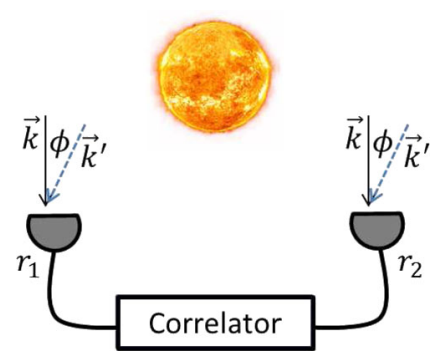


$E_{k} e^{i \vec{k} \cdot \vec{r}}$ and $E_{k^{\prime}} e^{i \vec{k}^{\prime} \cdot \vec{r}}$, the total amplitudes at $r_{1}$ and $r_{2}$ can be written as

$$
\begin{aligned}
& E\left(\vec{r}_{1}\right)=E_{k} e^{i \vec{k} \cdot \vec{r}_{1}}+E_{k^{\prime}} e^{i \vec{k}^{\prime} \cdot \vec{r}_{1}}, \\
& E\left(\vec{r}_{2}\right)=E_{k} e^{i \vec{k} \cdot \vec{r}_{2}}+E_{k^{\prime}} e^{i \vec{k}^{\prime} \cdot \vec{r}_{2}} .
\end{aligned}
$$

The intensities at $r_{1}$ and $r_{2}$ can be measured with two detectors. The signals which are proportional to the intensities are multiplied and integrated in a correlator. And finally, this can be described as

$$
C=\left\langle E^{*}\left(\vec{r}_{1}\right) E^{*}\left(\vec{r}_{2}\right) E\left(\vec{r}_{1}\right) E\left(\vec{r}_{2}\right)\right\rangle=\left\langle\left(\left|E_{k}\right|^{2}+\left|E_{k^{\prime}}\right|^{2}\right)^{2}\right\rangle+2\left\langle\left|E_{k}\right|^{2}\right\rangle\left\langle\left|E_{k^{\prime}}\right|^{2}\right\rangle \cos \left(k r_{0} \phi\right)
$$

where, $k=|\vec{k}|=\left|\vec{k}^{\prime}\right|$ and $r_{0}=\left|\vec{r}_{1}-\vec{r}_{2}\right|$ is the magnitude of the vectorial distance between the two detectors. By varying the separation of the detectors, we can get the angle between the two rays from Eq. (2), and from that, we can get the size of the star. As the intensity of each light ray is proportional to the photon number, the correlation function in Eq. (2) can be written as

$$
C=\left\langle\hat{n}_{k}^{2}\right\rangle+\left\langle\hat{n}_{k^{\prime}}^{2}\right\rangle+2\left\langle\hat{n}_{k}\right\rangle\left\langle\hat{n}_{k^{\prime}}\right\rangle\left[1+\cos \left(k r_{0} \phi\right)\right]
$$

where $\left\langle\hat{n}_{k}\right\rangle$ and $\left\langle\hat{n}_{k^{\prime}}\right\rangle$ are the mean photon number of the two light.

The noise is defined as $\Delta \hat{C}=\sqrt{\left\langle\hat{C}^{2}\right\rangle-\langle\hat{C}\rangle^{2}}$. In the HBT interferometer, $\hat{C}=\hat{I}\left(\vec{r}_{1}\right) \hat{I}\left(\vec{r}_{2}\right)$, and then we obtain the noise function as shown in the below

$$
\begin{aligned}
(\Delta \hat{C})^{2}= & \left\langle\hat{n}_{k}^{4}\right\rangle-\left\langle\hat{n}_{k}^{2}\right\rangle^{2}+8\left\langle\hat{n}_{k}^{3}\right\rangle\left\langle\hat{n}_{k^{\prime}}\right\rangle-4\left\langle\hat{n}_{k}^{2}\right\rangle\left\langle\hat{n}_{k}\right\rangle\left\langle\hat{n}_{k^{\prime}}\right\rangle+8\left\langle\hat{n}_{k}\right\rangle\left\langle\hat{n}_{k^{\prime}}^{3}\right\rangle \\
& -4\left\langle\hat{n}_{k}\right\rangle\left\langle\hat{n}_{k^{\prime}}\right\rangle\left\langle\hat{n}_{k^{\prime}}^{2}\right\rangle+16\left\langle\hat{n}_{k}^{2}\right\rangle\left\langle\hat{n}_{k^{\prime}}^{2}\right\rangle+\left\langle\hat{n}_{k^{\prime}}^{4}\right\rangle-\left\langle\hat{n}_{k^{\prime}}^{2}\right\rangle^{2}-6\left\langle\hat{n}_{k}\right\rangle^{2}\left\langle\hat{n}_{k^{\prime}}\right\rangle^{2} \\
& +4\left[2\left(\left\langle\hat{n}_{k}^{3}\right\rangle\left\langle\hat{n}_{k^{\prime}}\right\rangle+2\left\langle\hat{n}_{k}^{2}\right\rangle\left\langle\hat{n}_{k^{\prime}}^{2}\right\rangle+\left\langle\hat{n}_{k}\right\rangle\left\langle\hat{n}_{k^{\prime}}^{3}\right\rangle\right)-\left\langle\hat{n}_{k}^{2}\right\rangle\left\langle\hat{n}_{k}\right\rangle\left\langle\hat{n}_{k^{\prime}}\right\rangle-2\left\langle\hat{n}_{k}\right\rangle^{2}\left\langle\hat{n}_{k^{\prime}}\right\rangle^{2}\right. \\
& \left.-\left\langle\hat{n}_{k}\right\rangle\left\langle\hat{n}_{k^{\prime}}\right\rangle\left\langle\hat{n}_{k^{\prime}}^{2}\right\rangle\right] \cos \left(k r_{0} \phi\right)+2\left(\left\langle\hat{n}_{k}^{2}\right\rangle\left\langle\hat{n}_{k^{\prime}}^{2}\right\rangle-2\left\langle\hat{n}_{k}\right\rangle^{2}\left\langle\hat{n}_{k^{\prime}}\right\rangle^{2}\right) \cos \left(2 k r_{0} \phi\right) .
\end{aligned}
$$

For the thermal state, we have

$$
\begin{aligned}
& \left\langle\hat{n}^{2}\right\rangle=2 n_{\mathrm{th}}^{2}+n_{\mathrm{th}}, \\
& \left\langle\hat{n}^{3}\right\rangle=n_{\mathrm{th}}\left(1+6 n_{\mathrm{th}}+6 n_{\mathrm{th}}^{2}\right), \\
& \left\langle\hat{n}^{4}\right\rangle=n_{\mathrm{th}}\left(1+14 n_{\mathrm{th}}+36 n_{\mathrm{th}}^{2}+24 n_{\mathrm{th}}{ }^{3}\right),
\end{aligned}
$$

where, $n_{\text {th }}$ is the mean photon number of the thermal state.

For the sake of bookkeeping, we represent the mean photon number of light rays with wave vectors, $\vec{k}$ and $\vec{k}^{\prime}$ as $\bar{n}$ and $\bar{m}$ respectively. Using this, the correlation function of the $\mathrm{HBT}$ interferometer is given by

$$
C=2 \bar{n}^{2}+\bar{n}+2 \bar{m}^{2}+\bar{m}+2 \bar{n} \bar{m}\left[1+\cos \left(k r_{0} \phi\right)\right]
$$

and, the noise is given by

$$
\begin{aligned}
(\Delta \hat{C})^{2}= & \bar{n}+13 \bar{n}^{2}+32 \bar{n}^{3}+20 \bar{n}^{4}+\bar{m}+13 \bar{m}^{2}+32 \bar{m}^{3}+20 \bar{m}^{4}+32 \bar{n} \bar{m} \\
& +76 \bar{n}^{2} \bar{m}+76 \bar{n} \bar{m}^{2}+40 \bar{n}^{3} \bar{m}+40 \bar{n} \bar{m}^{3}+70 \bar{n}^{2} \bar{m}^{2}+2 \bar{n} \bar{m}(1+2 \bar{n}
\end{aligned}
$$


Figure 2 Schematic diagram of an ideal OPA. It consists of a pump beam and two input modes denoted by annihilation operators, $\hat{a}_{\text {in }}$ and $\hat{b}_{\text {in }}$, incident on the OPA. The $\hat{a}_{\text {out }}$ and $\hat{b}_{\text {out }}$ are the two output modes

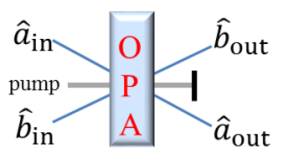

$$
\begin{aligned}
& +2 \bar{m}+2 \bar{n} \bar{m}) \cos \left(2 k r_{0} \phi\right)+4 \bar{n} \bar{m}\left(6+15 \bar{n}+10 \bar{n}^{2}+15 \bar{m}+10 \bar{m}^{2}\right. \\
& +8 \bar{n} \bar{m}) \cos \left(k r_{0} \phi\right) .
\end{aligned}
$$

\section{Model of an optical parametric amplifier}

An optical parametric amplifier, abbreviated as OPA, is a light source that emits light of variable wavelengths by an optical parametric amplification process. As depicted in Fig. 2, an OPA is a device with two input modes, $\hat{a}_{\text {in }}$ and $\hat{b}_{\text {in }}$ and two output modes, $\hat{a}_{\text {out }}$ and $\hat{b}_{\text {out }}$, which performs the mode evolution as [23-25]

$$
\left(\begin{array}{l}
\hat{a}_{\text {out }} \\
\hat{b}_{\text {out }}^{\dagger}
\end{array}\right)=\hat{T}_{\text {OPA }}\left(\begin{array}{l}
\hat{a}_{\text {in }} \\
\hat{b}_{\text {in }}^{\dagger}
\end{array}\right),
$$

where

$$
\hat{T}_{\mathrm{OPA}}=\left(\begin{array}{cc}
\mu & v \\
v^{*} & \mu
\end{array}\right)
$$

with, $\mu=\cosh g, v=e^{i \theta} \sinh g$, and $\theta$ and $g$ is the phase shift and parametrical strength in the OPA.

We can always choose the parameters in the propagation process in Eq. (8). Without loss of generality, assuming, $\theta=0$, we can write the relation between the input and output modes as

$$
\begin{aligned}
& \hat{a}_{\text {out }}=\hat{a}_{\text {in }} \cosh g+\hat{b}_{\text {in }}^{\dagger} \sinh g, \\
& \hat{b}_{\text {out }}^{\dagger}=\hat{a}_{\text {in }} \sinh g+\hat{b}_{\text {in }}^{\dagger} \cosh g .
\end{aligned}
$$

If we inject a thermal state to the upper mode and a vacuum state to the lower mode, then after the propagation, we have

$$
\begin{aligned}
\left\langle\hat{n}_{\text {out }}\right\rangle= & \mu^{2}\left\langle\hat{n}_{\text {in }}\right\rangle+v^{2}, \\
\left\langle\hat{n}_{\text {out }}^{2}\right\rangle= & \mu^{4}\left\langle\hat{n}_{\text {in }}^{2}\right\rangle+3 \mu^{2} v^{2}\left\langle\hat{n}_{\text {in }}\right\rangle+\mu^{2} v^{2}+v^{4}, \\
\left\langle\hat{n}_{\text {out }}^{3}\right\rangle= & \mu^{6}\left\langle\hat{n}_{\text {in }}^{3}\right\rangle+6 \mu^{4} v^{2}\left\langle\hat{n}_{\text {in }}^{2}\right\rangle+4 \mu^{4} v^{2}\left\langle\hat{n}_{\text {in }}\right\rangle+7 \mu^{2} v^{4}\left\langle\hat{n}_{\text {in }}\right\rangle \\
& +\mu^{4} v^{2}+4 \mu^{2} v^{4}+v^{6}, \\
\left\langle\hat{n}_{\text {out }}^{4}\right\rangle= & \mu^{8}\left\langle\hat{n}_{\text {in }}^{4}\right\rangle+10 \mu^{6} v^{2}\left\langle\hat{n}_{\text {in }}^{3}\right\rangle+10 \mu^{6} v^{2}\left\langle\hat{n}_{\text {in }}^{2}\right\rangle+25 \mu^{4} v^{4}\left\langle\hat{n}_{\text {in }}^{2}\right\rangle \\
& +\left(30 \mu^{4} v^{4}+5 \mu^{6} v^{2}+15 \mu^{2} v^{6}\right)\left\langle\hat{n}_{\text {in }}\right\rangle+11 \mu^{4} v^{4}+\mu^{6} v^{2} \\
& +11 \mu^{2} v^{6}+v^{8},
\end{aligned}
$$

where, $\mu=\cosh g, v=\sinh g$ and $\hat{n}_{\text {in }}=\hat{a}_{\text {in }}^{\dagger} \hat{a}_{\text {in }}$. 
Figure 3 Schematic diagram of our improved HBT interferometer. There are two OPAs placed at $r_{1}$ and $r_{2}$. One input of the OPAs is the star light and the other input is the vacuum state (VAC). Two outputs given by, $\hat{a}_{1, \text { out }}$ and $\hat{a}_{2, \text { out }}$, are detected and the signals are injected into the correlator

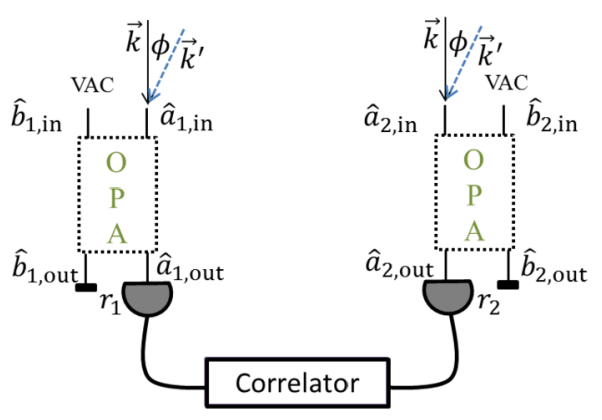

From Eq. (10), we know that an OPA can boost the input photon number. Thus, we can apply OPAs to the HBT interferometer to obtain an amplified correlation signal we need.

\section{Optical parametric amplified HBT interferometer}

Our improved interferometric scheme is depicted in Fig. 3. We place two OPAs at $r_{1}$ and $r_{2}$ and let the starlight pass through the OPAs first. The notations, $\hat{a}_{j \text {,in }}\left(\hat{b}_{j \text {,in }}\right), \hat{a}_{j, \text { out }}\left(\hat{b}_{j \text {,out }}\right)$, corresponds to $\hat{a}_{\text {in }}\left(\hat{b}_{\text {in }}\right), \hat{a}_{\text {out }}\left(\hat{b}_{\text {out }}\right)$ in Fig. 2 , where $j=1,2$. The starlight is injected in the

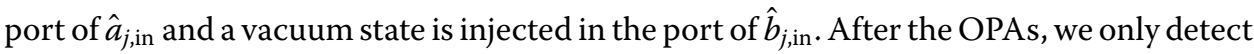
the output of $\hat{a}_{j \text {,in }}$ and then combine the two output signals into the correlator. With the property that OPA can boost the input photon number, we obtain an amplified correlation function.

Next, we calculate the correlation function and the noise of the new system. Using Eq. (11), we can obtain the forms of $\left\langle\hat{n}_{k, \text { out }}^{l}\right\rangle$ and $\left\langle\hat{n}_{k^{\prime}, \text { out }}^{l}\right\rangle(l=1,2,3,4)$ after passing through the OPAs. Plugging these new values into the correlation function and the noise function, the correlation function reduces to

$$
\begin{aligned}
C_{\mathrm{OPA}}= & \mu^{4}\left(2 \bar{n}^{2}+\bar{m}+2 \bar{m}^{2}\right)+\mu^{2} v^{2}(2+3 \mu+3 v)+\mu^{4} \bar{n} \\
& +2\left(\mu^{2} \bar{n}+v^{2}\right)\left(\mu^{2} \bar{m}+v^{2}\right)\left[1+\cos \left(k r_{0} \phi\right)\right],
\end{aligned}
$$

and, the noise reduces to

$$
\begin{aligned}
\left(\Delta \hat{C}_{\mathrm{OPA}}\right)^{2}= & \mu^{8}\left(\bar{n}+13 \bar{n}^{2}+32 \bar{n}^{3}+20 \bar{n}^{4}+\bar{m}+13 \bar{m}^{2}+32 \bar{m}^{3}+20 \bar{m}^{4}+32 \bar{n} \bar{m}\right. \\
& \left.+76 \bar{n}^{2} \bar{m}+76 \bar{n} \bar{m}^{2}+70 \bar{n}^{2} \bar{m}^{2}+40 \bar{n} \bar{m}^{3}+40 \bar{n}^{3} \bar{m}\right)+\mu^{6} v^{2}(2+55 \bar{n} \\
& +146 \bar{n}^{2}+88 \bar{n}^{3}+55 \bar{m}+146 \bar{m}^{2}+88 \bar{m}^{3}+240 \bar{n} \bar{m}+184 \bar{n}^{2} \bar{m} \\
& \left.+184 \bar{n} \bar{m}^{2}\right)+\mu^{4} v^{4}\left(50+215 \bar{n}+151 \bar{n}^{2}+211 \bar{m}+151 \bar{m}^{2}+248 \bar{n} \bar{m}\right) \\
& +30 v^{8}+\mu^{2} v^{6}(101+109 \bar{n}+113 \bar{m})+4 \cos \left(k r_{0} \phi\right)\left(\mu^{2} \bar{n}+v^{2}\right)\left(\mu^{2} \bar{m}\right. \\
& \left.+v^{2}\right)\left[\left(6+15 \bar{n}+10 \bar{n}^{2}+15 \bar{m}+10 \bar{m}^{2}+8 \bar{n} \bar{m}\right) \mu^{4}+(18+13 \bar{n}\right. \\
& \left.+13 \bar{m}) \mu^{2} v^{2}+4 v^{4}\right]+2 \cos \left(2 k r_{0} \phi\right)\left(\mu^{2} \bar{n}+v^{2}\right)\left(\mu^{2} \bar{m}+v^{2}\right)[(1 \\
& \left.+2 \bar{n}+2 \bar{m}+2 \bar{n} \bar{m}) \mu^{4}+2 \mu^{2} v^{2}-v^{4}\right] .
\end{aligned}
$$

Comparing Eq. (12) with Eq. (6), we can see that the signal amplitude of the correlation function is increased by a factor of $\left(\bar{n} \cosh ^{2} g+\sinh ^{2} g\right)\left(\bar{m} \cosh ^{2} g+\sinh ^{2} g\right) / \bar{n} \bar{m}$. We plot 

interferometer scheme to signal of the conventional HBT interferometer scheme as a function of mean number of photons $\bar{n}$ in light rays $\vec{k}$ and $\vec{k}^{\prime}$ when $g=2$. The red line corresponds to the new scheme without noise. The orange dotted line and the purple dotted line correspond to the new schemes with photon loss $L=0.5$ and photon loss $L=0.1$, respectively. The blue dotted line and the green dotted line correspond respectively to the new schemes with beam splitter transmissivity $T=50 \%$ and $\mathrm{T}=90 \%$ when the photon number of thermal noise is $N=0.1$. Note that the orange line and the blue line are overlapped, and the purple and green are, too

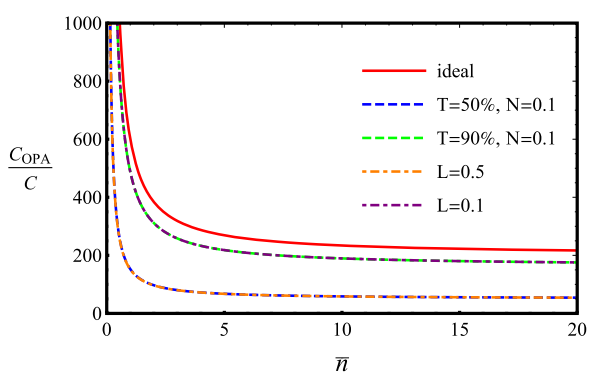

Figure 4 Ratios of signal of the improved HBT

Figure 5 Ratios of SNR of the improved HBT interferometer scheme SNR2 to SNR of the conventional HBT interferometer scheme SNR1 as a function of mean number of photons $\bar{n}$ in light rays $\vec{k}$ and $\vec{k}^{\prime}$ when $g=2$. The red line corresponds to the new scheme without noise. The orange dotted line and the purple dotted line correspond to the new schemes with photon loss $L=0.5$ and photon loss $\mathrm{L}=0.1$, respectively. The blue dotted line and the green dotted line correspond respectively to the new schemes with beam splitter transmissivity

$\mathrm{T}=50 \%$ and $\mathrm{T}=90 \%$ when the photon number of

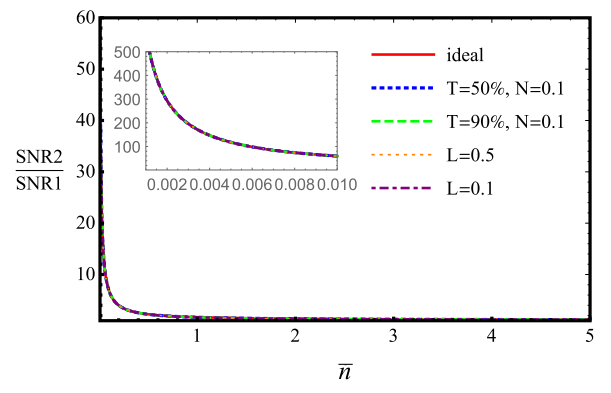

thermal noise is

$\mathrm{N}=0.1$. Note that all the lines are indistinguishably aligned

the ratio of the signals of the two interferometer systems as the red curve shown in Fig. 4. The parametrical strength $g$ usually ranges from 0 to 3 . In this figure, we take $g=2$ as an example and assume that both light sources have the same mean photon number. We see it would be at least a 200 times increase in the signal.

Once we have the signals and noises of both systems, we can compare the performance of these two systems. We use SNR as a indication of the system performance. As we discussed earlier, the signal is increased by the OPAs but what about SNR? We define the SNR as

$$
\mathrm{SNR}=C / \Delta \hat{C} .
$$

With the correlation functions and noise functions, the SNR ratio of the two systems can be obtained. Figure 5 shows the ratios of SNR of the improved HBT interferometer scheme to SNR of the conventional HBT interferometer scheme as a function of mean number of photons. The red curve in Fig. 5, is the ratio of the SNRs of the two systems as a function of the mean photon number when $k r_{0} \phi=2 p \pi$ ( $p$ is an integer). From the red curve, we see that the ratio of SNRs is 1.66 when $\bar{n} \approx 1$, which means the SNR increases by about 66 percent with the use of the OPAs. Moreover, if $\bar{n}$ is much smaller, such as $\bar{n}=1.5 \times 10^{-3}$, then the SNR increases by 400 percent. As the function of the curve is too complicated, we use curve fitting to get an approximated function which is

$$
\frac{\mathrm{SNR} 2}{\mathrm{SNR} 1}=1.082+\frac{0.584}{\bar{n}} .
$$


From the above equation, one can estimate the value of SNR increased for an arbitrary small $\bar{n}$.

One can see all the discussion above is based on ideal situations. However, for any realistic model, one must consider the effect of noise, such as photon loss and thermal noise which are the most common factors. In the following, we will discuss the lossy models of the optical parametric amplified HBT interferometer caused by photon loss and thermal noise.

There are two mechanisms: photon loss to the environment occurring inside the interferometer and photon loss at the detectors, lead to the first lossy model. Both of the two processes can be modeled by placing a virtual beam splitter inside the interferometer. One input of the beam splitter is the state of the propagating mode in the interferometer arm and the other input is a vacuum state. In this case, the operator $\hat{a}_{j, \text { out }}$ in Fig. 3 becomes

$$
\hat{a}_{j, \text { out }}=\sqrt{1-\mathrm{L}}\left(\mu \hat{a}_{j, \text { in }}+v \hat{b}_{j, \text { in }}^{\dagger}\right)+\sqrt{\mathrm{L}} \hat{v}
$$

where $\mathrm{L}$ is the photon loss $(0 \leq \mathrm{L} \leq 1)$ and $\hat{v}$ is the annihilation operator of vacuum state. Then after the whole propagation through the interferometer, we get ratios of the two systems shown as the orange curve with photon loss $L=0.5$ and the purple curve with photon loss $\mathrm{L}=0.1$ in Fig. 4 and Fig. 5 . One can see that the photon loss does not have any effect on the SNR but have slight influence on the signals. Although the ratio of signals of the two interferometer schemes becomes smaller with the increasing of the photon loss, at least, it could be increased by one orders of magnitude. The reason that photon loss does not decrease the SNR is straightforward according to our new interferometric scheme has better result in weak light.

Then, we will discuss the interaction with thermal noise. This process can be accomplished much in the same way as the photon loss model. We also place beam splitters inside the interferometer, but the vacuum input is replaced by a thermal state. The thermal photon noise is approximately $\mathrm{N}=10^{-20}$ at room temperature, and $\mathrm{N}=1$ can be obtained in microwave frequency [26]. In our case, we take the thermal photon noise as $\mathrm{N}=0.1$. We get the ratios shown as the blue curve when beam splitter transmissivity $\mathrm{T}=50 \%$, thermal noise $\mathrm{N}=0.1$ and the green curve with transmissivity $\mathrm{T}=90 \%$, thermal noise $\mathrm{N}=0.1$ in Fig. 4 and Fig. 5. Easily, one can see thermal noise has the same effect as the photon loss on the condition that the beam splitter has the same transmissivity in Fig. 4. Remarkably, the ratio of the two SNRs remains unchanged for both propagation loss and detector inefficiency as one can see in Fig. 5 that all the lines are indistinguishably aligned.

\section{Conclusion}

In conclusion, we have proposed a new interferometric scheme that combines OPAs with HBT interferometers. In this scheme, instead of measuring the intensity of the starlight directly, we let the starlight go through the two OPAs first, amplifying the correlation signal by a factor of $\left(\bar{n} \cosh ^{2} g+\sinh ^{2} g\right)^{2} / \bar{n}^{2}$, where $\bar{n}$ is the mean photon number of the star light. Although the noise also increases in our new scheme, the theoretical analysis shows that, the participation of OPAs leads to an improvement of the SNR of a factor $1.082+$ $0.584 / \bar{n}$ compared to the conventional SNR in conventional HBT interferometer. One can see, for the case of weak starlight, which means that the mean photon number is very small, the SNR is much higher than that of conventional HBT interferometer. In astronomy, the 
intensity of light that reaches the detector from the majority of stars is very weak, thus, our scheme will be very helpful. Moreover, the idea that SNR can be amplified by OPAs may also find applications in quantum remote sensing and long-distance quantum imaging, where the light passed through the object is weak after a long distance propagation.

\author{
Acknowledgements \\ This work was accepted shortly after the tragic death of one of the authors, Jonathan P. Dowling. We acknowledge his \\ helpful guidance and his insight, and we are forever grateful that we were able to work with him closely. \\ Funding \\ XM acknowledges financial support from CSC, National Natural Science Foundation of China (Grant No. 61575180, \\ $61701464,11475160,61640009$ ) and the Natural Science Foundation of Shandong Province (Grants No. ZR2014AQ026 \\ and No. ZR2014AM023). CY would like to acknowledge support from Economic Development Assistantship from \\ Louisiana State University System Board of Regents. SA, HL and JPD would like to acknowledge support from the Air \\ Force office of Scientific Research, the Army Research office, the Defense Advanced Research Projects Agency and the \\ National Science Foundation.
}

\title{
Abbreviations
}

HBT, Hanbury Brownand and Twiss; OPA, optical parametric amplifier; SNR, signal-to-noise ratio.

\section{Availability of data and materials}

Not applicable.

\section{Competing interests}

The authors declare that they have no competing interests.

\section{Authors' contributions}

XM implemented numerical simulations and was a major contributor in writing the manuscript. CY contributed to the initiation of the research. SA contributed to writing and revising the manuscript. YG contributed to the comment and revision of the manuscript. OSML contributed to the formulation of the optimisation problem, comment and revision of the manuscript. JPD contributed to the comment and revision of the manuscript. HL contributed to the initiation of the research, introduction, comment and revision of the paper. All authors read and approved the final manuscript.

\section{Author details}

'College of Mathematical and Physical Sciences, Qingdao University of Science and Technology, Qingdao, China. ${ }^{2}$ Hearne Institute for Theoretical Physics and Department of Physics \& Astronomy, Louisiana State University, Baton Rouge, USA. ${ }^{3}$ Quantum Photonics Laboratory, Department of Physics and Astronomy, Louisiana State University, Baton Rouge, USA. ${ }^{4}$ Department of Physics, Ocean University of China, Qingdao, China. ${ }^{5} \mathrm{NYU}$-ECNU Institute of Physics, NYU Shanghai, Shanghai, China. ${ }^{6}$ CAS-Alibaba Quantum Computing Laboratory, CAS Center for Excellence in Quantum Information and Quantum Physics, University of Science and Technology of China, Shanghai, China.

\section{Publisher's Note}

Springer Nature remains neutral with regard to jurisdictional claims in published maps and institutional affiliations.

Received: 5 February 2020 Accepted: 15 June 2020 Published online: 29 June 2020

\section{References}

1. Davis J. Stellar angular diameter measurements by interferometry. In: Bedding TR, Booth AJ, Davis J, editors. Fundamental stellar properties: the interaction between observation and theory. Dordrecht: Springer; 1997. p. 31-8.

2. Hanbury Brown R, Twiss RQ. A test of a new type of stellar interferometer on sirius. Nature. 1956;178(4541):1046-8.

3. Hanbury Brown R, Twiss RQ. Correlation between photons in two coherent beams of light. Nature. 1956;177(4497):27-9.

4. Glauber RJ. Coherent and incoherent states of the radiation field. Phys Rev. 1963;131(6):2766.

5. Glauber RJ. The quantum theory of optical coherence. Phys Rev. 1963;130(6):2529.

6. Mandel L, Wolf E. Coherence properties of optical fields. Rev Mod Phys. 1965;37(2):231-87.

7. Saleh BEA, Stole D, Teich MC. Coherence and photon statistics for optical fields generated by Poisson random emissions. Phys Rev A. 1983;27(1):360-74.

8. Singer A, Lorenz U, Sorgenfrei F, Gerasimova N, Gulden J, Yefanov OM, Kurta RP, Shabalin A, Dronyak R, Treusch R, Kocharyan V, Weckert E, Wurth W, Vartanyants IA. Hanbury brown-twiss interferometry at a free-electron laser. Phys Rev Lett. 2013;111(3):034802.

9. Magaña-Loaiza OS, Mirhosseini M, Cross RM, Rafsanjani SMH, Boyd RW. Hanbury brown and twiss interferometry with twisted light. Sci Adv. 2016;2(4):1501143.

10. Bai B, Zhou Y, Liu R, Zheng H, Wang Y, Li F, Xu Z. Hanbury brown-twiss effect without two-photon interference in photon counting regime. Sci Rep. 2017.

11. Baym G. The physics of hanbury brown-twiss intensity interferometry: from stars to nuclear collisions. Acta Phys Pol B. 1998;29:1839-84.

12. Jeltes T, McNamara JM, Hogervorst W, Vassen W, Krachmalnicoff $V$, Schellekens M, Perrin A, Chang H, Boiron D, Aspect A, Westbrook Cl. Comparison of the hanbury brown-twiss effect for bosons and fermions. Nature. 2007;445:402-5. 
13. Campagnano G, Zilberberg O, Gornyi IV, Feldman DE, Potter AC, Gefen Y. Hanbury brown-twiss interference of anyons. Phys Rev Lett. 2012;109(10):106802.

14. Silva B, Sánchez Muñoz C, Ballarini D, González-Tudela A, de Giorgi M, Gigli G, West K, Pfeiffer L, del Valle E, Sanvitto D, Laussy FP. The colored hanbury brown-twiss effect. Sci Rep. 2016;6:37980.

15. Mirhosseini M, Magaña-Loaiza OS, Chen C, Hashemi Rafsanjani SM, Boyd RW. Wigner distribution of twisted photons. Phys Rev Lett. 2016;116:130402.

16. Schellekens M, Hoppeler R, Perrin A, Viana Gomes J, Boiron D, Aspect A, Westbrook CI. Hanbury brown twiss effect for ultracold quantum gases. Science. 2005;310(5748):648-51.

17. Hassinen T, Tervo J, Setälä T, Friberg AT. Hanbury brown-twiss effect with electromagnetic waves. Opt Express. 2011;19(16):15188-95.

18. Silva EF, Barbosa ALR, Ramos J. Parity and time-reversal symmetry in the hanbury brown-twiss effect. Europhys Lett. 2017;117(1):14001.

19. You C, Quiroz-Juarez MA, Lambert A, Bhusal N, Dong C, Perez-Leija A, Javaid A, León-Montiel R de J. Identification of light sources using machine learning. Appl Phys Rev. 2020;7:021404.

20. Magaña-Loaiza OS, Boyd RW. Quantum imaging and information. Rep Prog Phys. 2019:82(12):124401.

21. You C, Nellikka AC, Leon ID, Magaña-Loaiza OS. Multiparticle quantum plasmonics. Nanophotonics. 2020;20190517.

22. Hanbury Brown R. The intensity interferometer: its application to astronomy. London: Taylor \& Francis; 1974

23. Yurke B, McCall SL, Klauder JR. Su(2) and su(1,1) interferometers. Phys Rev A. 1986;33:4033-54.

24. Ma X, You C, Adhikari S, Matekole ES, Glasser RT, Lee H, Dowling JP. Sub-shot-noise-limited phase estimation via su $(1,1)$ interferometer with thermal states. Opt Express. 2018;26:18492-504.

25. You C, Adhikari S, Ma X, Sasaki M, Takeoka M, Dowling JP. Conclusive precision bounds for su(1,1) interferometers. Phys Rev A. 2019;99:042122.

26. Zhang J-D, Zhang Z-J, Cen L-Z, You C, Adhikari S, Dowling JP, Zhao Y. Orbital-angular-momentum-enhanced estimation of sub-Heisenberg-limited angular displacement with two-mode squeezed vacuum and parity detection. Opt Express. 2018;26(13):16524-34.

\section{Submit your manuscript to a SpringerOpen ${ }^{\circ}$ journal and benefit from:}

- Convenient online submission

- Rigorous peer review

- Open access: articles freely available online

- High visibility within the field

- Retaining the copyright to your article

Submit your next manuscript at $\boldsymbol{\nabla}$ springeropen.com 